Pacific

Journal of

Mathematics

LONG-TIME EXISTENCE OF MEAN CURVATURE FLOW WITH EXTERNAL FORCE FIELDS

HUaI-Yu Jian AND YANNAN LiU

Volume $234 \quad$ No. 2

February 2008 


\title{
LONG-TIME EXISTENCE OF MEAN CURVATURE FLOW WITH EXTERNAL FORCE FIELDS
}

\author{
HUAI-YU JiAN AND YANNAN LIU
}

\begin{abstract}
We study the evolution of submanifolds moving by mean curvature and an external force field. We prove flow has a long-time smooth solution for all time under almost optimal conditions. Those conditions are that the second fundamental form on the initial submanifolds is not too large, the external force field and all of it derivatives are bounded, and the field is convex with its eigenvalues satisfying a pinch inequality.
\end{abstract}

\section{Introduction}

We study the flow

$$
\frac{d F_{t}}{d t}=-\left(H_{\alpha}-\omega_{\alpha}\right) e_{\alpha} \equiv-f_{\alpha} e_{\alpha}
$$

where $F_{t}:=F(\cdot, t): M^{n} \rightarrow \mathbb{R}^{n+k}$ is a family of smooth immersions, $M_{t}=F_{t}(M)$, $M$ is a compact oriented submanifold in $\mathbb{R}^{n+k}, H$ is the mean curvature vector of $M_{t}$ with respect to the unit normal field $e_{\alpha}$ for $\alpha=n+1, \ldots, n+k, \omega$ is a given smooth function in $\mathbb{R}^{n+k}, \bar{\nabla} \omega$ is the standard gradient field of $\omega$ in $\mathbb{R}^{n+k}$, and $\omega_{\alpha} \equiv\left\langle\bar{\nabla} \omega, e_{\alpha}\right\rangle$.

This flow generalizes the well-known mean curvature flow, that is, the case $\omega \equiv$ const, and it comes directly from the study of the Ginzburg-Landau vortex. As was shown in [Jian and Xu 2003; Jian and Liu 2006], there are two models that both reduce to the Ginzburg-Landau system of parabolic equations

$$
\frac{\partial V_{\varepsilon}}{\partial t}=\bar{\Delta} V_{\varepsilon}+\bar{\nabla} \omega \bar{\nabla} V_{\varepsilon}+A V_{\varepsilon}+\frac{B V_{\varepsilon}}{\varepsilon^{2}}\left(1-\left|V_{\varepsilon}\right|^{2}\right)
$$

in $\mathbb{R}^{m} \times(0, \infty)$, where $\varepsilon$ is a small positive parameter and $\omega, A$, and $B$ are known functions. One is a simple equation simulating inhomogeneous type $\Pi$ superconducting materials [Chapman and Richardson 1997], and the other simulates threedimensional superconducting thin films having variable thickness [Chapman et al.

MSC2000: primary 58J35, 53A07, 35K45; secondary 53A10, 35K55, 58J15.

Keywords: parabolic equation, mean curvature flow, maximum principle for tensors.

Supported by the Natural Science Foundation of China (No. 10631020) and the Grant for PhD Programs of the Ministry of Education of China. 
1996]. An important problem in Ginzburg-Landau superconductors is to study the vortex dynamics, that is, the convergence of $V_{\varepsilon}$ and of their zero points (which, roughly, are called vortexes) as $\varepsilon \rightarrow 0$.

When $m=2$ and the initial vortex consists of finite isolated points, it was proved in [Jian and Xu 2003; Jian and Song 2001; Lin 1996] that the vortex dynamics of the Dirichlet problem for (1-2) is described by the ODE system

$$
\frac{\partial x}{\partial t}=-\bar{\nabla} \omega(x)
$$

When $m \geq 2$ and the initial vortex consists of a filament or even a codimension $k$ submanifold, Jian and Liu [2006] proved that as $\varepsilon \rightarrow 0$, the vortex of Cauchy problem for (1-2) is approximated by the evolution of the initial vortex according to flow (1-1) on the time internal in which the flow is smooth. Similar results were obtained for Neumann problem in [Lin 1998] and for case of $\bar{\nabla} \omega=0$ in [Jerrard and Soner 1999; Lin 1998]. Therefore, it is important in physics to consider the long-time existence of the flow (1-1).

On the other hand, mean curvature flow has been strongly studied in recent decades. It is well known that the flow must blow up in finite time unless the initial submanifolds are graphic; see [Chen and Li 2001; 2004; Huisken 1984; 1990; Jian 2006; Jian et al. 2005; Smoczyk and Wang 2002; Wang 2002] for details. Hence it is natural to ask, for what kind of functions does $\omega$ have long-time existence?

Higher codimension mean curvature flow, that is, (1-1) without an external force field, has been studied in [Chen and Li 2001; 2004; Jian 2006; Smoczyk and Wang 2002; Wang 2002]. There have also been many studies of mean curvature flow for hypersurfaces; see [Huisken 1984; 1990; Jian et al. 2005] for example. All those papers show that mean curvature flow must blow up so that a singularity occurs in finite time, unless the initial surfaces are entire graphs or graphic submanifolds.

We concentrate on the long-time existence of (1-1). Here is our main result:

Theorem 1.1. If there exist positive constants $C, C_{3}, \bar{\lambda}, \underline{\lambda}$ with $\bar{\lambda}<2 \underline{\lambda}$ such that

(i) $\underline{\lambda}|\xi|^{2} \leq \bar{\nabla}_{i j}^{2} \omega(x) \xi_{i} \xi_{j} \leq \bar{\lambda}|\xi|^{2}$ and $\left|\bar{\nabla}^{3} \omega(x)\right| \leq C_{3}$ for all $\xi \in \mathbb{R}^{n+k}$ and for all $x \in \mathbb{R}^{n+k}$;

(ii) $|A|^{2}<C$ on $M_{0}$;

(iii) the constants $C, C_{3}, \bar{\lambda}$, and $\underline{\lambda}$ satisfy $5 C^{3 / 2}+C_{3}+(\bar{\lambda}-2 \underline{\lambda}) C^{1 / 2}<0$; and

(iv) $\left|\bar{\nabla}^{i} \omega(x)\right|$ is uniformly bounded for all $x \in \mathbb{R}^{n+k}$ and $i=1,2, \ldots$,

then the flow (1-1) has a smooth solution for all time $t \in[0, \infty)$.

Throughout this paper, flow (1-1) is denoted by (1-1)' in the case $k=1$, that is, the case of hypersurfaces. 
Theorem 1.2. Suppose that the assumptions of Theorem 1.1 are satisfied except that (1-1) is replaced by (1-1)' and (iii) is replaced by

(iii)' the constants $C, C_{3}, \bar{\lambda}$, and $\underline{\lambda}$ satisfy $C^{3 / 2}+C_{3}+(\bar{\lambda}-2 \underline{\lambda}) C^{1 / 2}<0$.

Then the flow (1-1)' has a smooth solution for all time $t \in[0, \infty)$.

Remark 1.3. From the proof of Theorems 1.1 and 1.2, we realize this: Instead of "for all $x \in \mathbb{R}^{n+k}$ ", it is sufficient to suppose that assumptions (i) and (iv) hold for "for all $x \in M_{t}$, where $M_{t}$ is any solution of (1-1) on any finite time interval $[0, T]$ ".

The following theorem generalizes the convexity preservation of mean curvature flow in [Huisken 1984].

Theorem 1.4. Let $T>0$, and let $M_{t}$ be a smooth solution of flow (1-1)' on the time interval $[0, T]$. If $\bar{\nabla}^{3} \omega \equiv 0$ and $M_{0}$ is convex, then $M_{t}$ is convex for all $t \in[0, T]$.

Physically, $\omega$ is a density function and actually has the form

$$
\omega=\frac{1}{2}\left(c_{1} x_{1}^{2}+\ldots+c_{n+1} x_{n+1}^{2}\right)
$$

for $c_{i}>0$, (see [Chapman and Richardson 1997] for example), but Theorems 1.1 and 1.2 cannot be applied directly to this special case, because $|\bar{\nabla} \omega|$ is not yet known to be bounded uniformly. However, we can give the long-time existence for this $\omega$ in the case of hypersurfaces.

Corollary 1.5. Suppose $\omega=\frac{1}{2}\left(c_{1} x_{1}^{2}+\ldots+c_{n+1} x_{n+1}^{2}\right)$, where $c_{i}$ are positive constants, and let $M=\max c_{i}$ and $m=\min c_{i}$. If $M<2 m$ and $|A|^{2}<2 m-M$ on $M_{0}$, then for any $T>0$ the flow (1-1)' has a smooth solution for all $t \in[0, T]$.

We point out that Corollary 1.5 generalizes [Liu and Jian 2007, Theorem 1.3], which studies (1-1)' for the special case $\omega=\frac{1}{2} c|x|^{2}$. That theorem also shows that flow (1-1)' must blow up in finite time either if $c<0$ or if $c>0$ and $|A|^{2}>c$ on $M_{0}$, which means that the both convexity of $\omega$, as in assumption (i), and the smallness of the initial $|A|^{2}$, as in assumptions (ii) and (iii)', are necessary. Also see Remark 3.4.

In Section 2, we will give notations and preliminaries. In Section 3, we give the proofs of Theorems 1.2 and 1.4 and Corollary 1.5. Finally, in Section 4, we prove Theorem 1.1.

\section{Preliminaries}

We use the following notations throughout. $\langle\cdot, \cdot\rangle$ denotes the usual inner product in $\mathbb{R}^{n+k}$. If $M$ is given as in Section 1 and $F$ denotes its parametrization in $\mathbb{R}^{n+k}$, 
the components of the metric $\left\{g_{i j}\right\}$ are given by

$$
g_{i j}(x)=\left\langle\frac{\partial F(x)}{\partial x_{i}}, \frac{\partial F(x)}{\partial x_{j}}\right\rangle \text { for } x \in M .
$$

Let $\nabla$ denote the Levi-Civita connection on $\mathrm{M}$, and let $\bar{\nabla}$ denote the standard gradient in $\mathbb{R}^{n+k}$. We use Latin letters $i, j, k, \cdots$ for tangent indices and use Greek letters $\alpha, \beta, \gamma, \cdots$ for normal ones. Repeated Latin indices are to be summed from 1 to $n$, and repeated Greek indices sum from 1 to $k$. Indices are raised and lowered by $g^{i j}$ and $g_{i j}$, respectively. We identify $V \in T_{x} M$ with $D F(V) \in T_{F(x)} \mathbb{R}^{n+k}$. We also use $\langle\cdot, \cdot\rangle$ to denote the scalar product on $M$ when there is no risk of confusion.

The second fundamental form in the direction $e_{\alpha}$, the norm of the second fundamental form, and the mean curvature on $M$ in the direction $e_{\alpha}$ are respectively

$$
h_{\alpha i j}(x)=-\left\langle e_{\alpha}, \nabla_{i} \nabla_{j} F\right\rangle, \quad|A|^{2}=g^{i j} g^{k l} h_{\alpha i k} h_{\alpha l j}, \quad \text { and } H_{\alpha}=g^{i j} h_{\alpha i j} .
$$

Let $R_{i j k l}$ denote the curvature tensor, let $R_{\beta \alpha j k}^{\perp}$ denote the normal curvature tensor, and recall Ricci's equation and Gauss's equation giving these curvatures on a submanifold of Euclidean space:

$$
R_{\alpha \beta i j}^{\perp}=h_{\alpha i k} h_{\beta j k}-h_{\alpha j k} h_{\beta i k} \quad \text { and } \quad R_{i j k l}=h_{\alpha i k} h_{\alpha j l}-h_{\alpha j k} h_{\alpha i l} .
$$

Of course, $R^{\perp}$ is zero for a hypersurface. Also, we can write the Weingarten equation and the Codazzi equation respectively as

$$
\begin{aligned}
\nabla_{i} e_{\beta} & =h_{\beta i}^{l} \nabla_{l} F+C_{i \beta}^{\gamma} e_{\gamma}, \\
h_{\alpha i k, j} & =h_{\alpha i j, k},
\end{aligned}
$$

where $C_{i \beta}^{\gamma}$ is the connection coefficient of the normal connection and $C_{i \beta}^{\gamma}=-C_{i \gamma}^{\beta}$. We will also use the following basic facts.

Proposition 2.1 [Huisken 1984; Schnürer and Smoczyk 2002]. For any hypersurface $M$ in $\mathbb{R}^{n+1}$, we have

$$
\begin{aligned}
\nabla_{i} \nabla_{j} F & =-h_{i j} v, \\
\nabla_{i} v & =h_{i}^{l} \nabla_{l} F, \\
\nabla_{k} h_{i j} & =\nabla_{j} h_{i k}, \\
\nabla_{i} \nabla_{j} H & =\triangle h_{i j}-H h_{i}^{l} h_{l j}+|A|^{2} h_{i j}, \\
2 h^{i j} \nabla_{i} \nabla_{j} H & =\triangle|A|^{2}-2|\nabla A|^{2}-2 Z,
\end{aligned}
$$

where $v$ is the outer normal vector of $M$,

$$
Z=H C_{0}-|A|^{4}, \quad \text { and } \quad C_{0}=g^{i j} g^{k l} g^{s t} h_{i k} h_{s j} h_{l t}=\operatorname{tr}\left(A^{3}\right) .
$$


Proposition 2.2 [Schnürer and Smoczyk 2002; Wang 2002]. Suppose flow (1-1) holds for $t \in[0, T)$ with $T \leq \infty$. Then

$$
\frac{d g_{i j}}{d t}=-2 f_{\alpha} h_{\alpha i j} \quad \text { and } \quad \frac{d g^{i j}}{d t}=2 f_{\alpha} h_{\alpha k l} g^{i k} g^{j l}
$$

hold on $M_{t}$ for all $t \in[0, T)$. In particular, for the flow (1-1)', we have

$$
\frac{d h_{i j}}{d t}=\nabla_{i} \nabla_{j} f-f h_{i}^{l} h_{l j} \quad \text { and } \quad \frac{d|A|^{2}}{d t}=2 h^{i j} \nabla_{i} \nabla_{j} f+2 f C_{0} .
$$

Here and below, we let $f \equiv f_{\alpha}=f_{1}$ for a hypersurface.

The following theorem for short time existence of (1-1) is well known from the theory of PDE and the technique of De Turk [Hamilton 1996].

Theorem 2.3 [Hamilton 1996]. The flow (1-1) is a system of qusilinear parabolic equations, and there exists a maximal time $0<T \leq \infty$ such that (1-1) admits a smooth solution on $[0, T)$.

\section{The case of hypersurfaces}

Here we prove Theorems 1.2 and 1.4 and Corollary 1.5. The key step is to calculate the evolution equations of $|A|^{2}$.

Proposition 3.1. Suppose flow (1-1)' holds for $t \in[0, T)$ with $T \leq \infty$, then this equation is satisfied on $M_{t}$ for all $t \in[0, T)$ :

$$
\begin{aligned}
& \frac{d|A|^{2}}{d t}=\Delta|A|^{2}-2|\nabla A|^{2}+2|A|^{4}-2 h^{i j}\left(\nabla_{i} \bar{\nabla}^{2} \omega\right)\left(\nabla_{j} F, v\right) \\
& +2|A|^{2} \bar{\nabla}^{2} \omega(v, v)-4 h^{i j} h_{j}^{l} \bar{\nabla}^{2} \omega\left(\nabla_{i} F, \nabla_{l} F\right)-\left\langle\bar{\nabla} \omega, \nabla|A|^{2}\right\rangle
\end{aligned}
$$

Proof. By the second of (2-10), we have $d|A|^{2} / d t=2 h^{i j} \nabla_{i} \nabla_{j} f+2 f C_{0}$. By the notation $f \equiv f_{\alpha}=f_{1}$ and using (2-8), we have

$$
\begin{aligned}
2 h^{i j} \nabla_{i} \nabla_{j} f & =2 h^{i j} \nabla_{i} \nabla_{j} H-2 h^{i j} \nabla_{i} \nabla_{j}\langle\bar{\nabla} \omega, v\rangle \\
& =\triangle|A|^{2}-2|\nabla A|^{2}-2 Z-2 h^{i j} \nabla_{i} \nabla_{j}\langle\bar{\nabla} \omega, v\rangle .
\end{aligned}
$$

It follows from (2-5) that

$$
\begin{aligned}
\nabla_{i} \nabla_{j}\langle\bar{\nabla} \omega, v\rangle= & \nabla_{i}\left(\left\langle\nabla_{j} \bar{\nabla} \omega, v\right\rangle+\left\langle\bar{\nabla} \omega, \nabla_{j} v\right\rangle\right) \\
= & \nabla_{i}\left(\left\langle\nabla_{j} \bar{\nabla} \omega, v\right\rangle+h_{j}^{l}\left\langle\bar{\nabla} \omega, \nabla_{l} F\right\rangle\right) \\
= & \left\langle\nabla_{i} \nabla_{j} \bar{\nabla} \omega, v\right\rangle+h_{i}^{l}\left\langle\nabla_{j} \bar{\nabla} \omega, \nabla_{l} F\right\rangle \\
& +h_{i}^{l}\left\langle\nabla_{j} \bar{\nabla} \omega, \nabla_{l} F\right\rangle+h_{j}^{l}\left\langle\bar{\nabla} \omega, \nabla_{i} \nabla_{l} F\right\rangle+\nabla_{i} h_{j}^{l}\left\langle\bar{\nabla} \omega, \nabla_{l} F\right\rangle
\end{aligned}
$$


Using (2-4) and (2-6), we obtain

$$
\begin{aligned}
\nabla_{i} \nabla_{j}\langle\bar{\nabla} \omega, v\rangle=\left\langle\nabla_{i} \nabla_{j} \bar{\nabla} \omega, v\right\rangle+h_{i}^{l}\left\langle\nabla_{j} \bar{\nabla} \omega, \nabla_{l} F\right\rangle \\
+h_{i}^{l}\left\langle\nabla_{j} \bar{\nabla} \omega, \nabla_{l} F\right\rangle+\left\langle\bar{\nabla} \omega, \nabla h_{i j}\right\rangle-h_{j}^{l} h_{i l}\langle\bar{\nabla} \omega, v\rangle,
\end{aligned}
$$

which implies

$$
\begin{aligned}
2 h^{i j} \nabla_{i} \nabla_{j}\langle\bar{\nabla} \omega, v\rangle=2 h^{i j}\left\langle\nabla_{i} \nabla_{j} \bar{\nabla} \omega, v\right\rangle+4 h^{i j} h_{j}^{l}\left\langle\nabla_{i} \bar{\nabla} \omega, \nabla_{l} F\right\rangle & \\
& +\left\langle\bar{\nabla} \omega, \nabla|A|^{2}\right\rangle-2 h^{i j} h_{j}^{l} h_{i l}\langle\bar{\nabla} \omega, v\rangle .
\end{aligned}
$$

This, together with (3-2), the second of (2-10), and the definitions of $f, C_{0}$, and $Z$, gives

$$
\begin{aligned}
\frac{d|A|^{2}}{d t}=\triangle|A|^{2}-2|\nabla A|^{2}+2|A|^{4} & -2 h^{i j}\left\langle\nabla_{i} \nabla_{j} \bar{\nabla} \omega, v\right\rangle \\
& -4 h^{i j} h_{j}^{l}\left\langle\nabla_{i} \bar{\nabla} \omega, \nabla_{l} F\right\rangle-\left\langle\bar{\nabla} \omega, \nabla|A|^{2}\right\rangle
\end{aligned}
$$

But

$$
\begin{aligned}
\left\langle\nabla_{i} \nabla_{j} \bar{\nabla} \omega, v\right\rangle= & \nabla_{i}\left(\left\langle\nabla_{j} \bar{\nabla} \omega, v\right\rangle\right)-\left\langle\nabla_{j} \bar{\nabla} \omega, \nabla_{i} v\right\rangle \\
= & \nabla_{i}\left(\bar{\nabla}^{2} \omega\left(\nabla_{j} F, v\right)\right)-\left\langle\nabla_{j} \bar{\nabla} \omega, \nabla_{i} v\right\rangle \\
= & \left(\nabla_{i} \bar{\nabla}^{2} \omega\right)\left(\nabla_{j} F, v\right) \\
& +\bar{\nabla}^{2} \omega\left(\nabla_{i} \nabla_{i} F, v\right)+\left\langle\nabla_{j} \bar{\nabla} \omega, \nabla_{i} v\right\rangle-\left\langle\nabla_{j} \bar{\nabla} \omega, \nabla_{i} v\right\rangle \\
= & \left(\nabla_{i} \bar{\nabla}^{2} \omega\right)\left(\nabla_{j} F, v\right)-h_{i j} \bar{\nabla}^{2} \omega(v, v),
\end{aligned}
$$

where we have used (2-4) for the last equality. Inserting this equality to (3-4), we get the desired equality (3-1).

Proposition 3.2. With the same assumptions as in Proposition 3.1, the second fundamental form satisfies this tensorial evolution equation on $M_{t}$ for all $t \in[0, T)$ :

$$
\begin{aligned}
& \frac{d h_{i j}}{d t}=\Delta h_{i j}-2 H h_{i}^{l} h_{l j}+|A|^{2} h_{i j}-\left(\nabla_{i} \bar{\nabla}^{2} \omega\right)\left(\nabla_{j} F, v\right) \\
&+h_{i j} \bar{\nabla}^{2} \omega(v, v)-h_{i}^{l} \bar{\nabla}^{2} \omega\left(\nabla_{j} F, \nabla_{l} F\right)-h_{i}^{l} \bar{\nabla}^{2} \omega\left(\nabla_{j} F, \nabla_{l} F\right) \\
&-\left\langle\bar{\nabla} \omega, \nabla h_{i j}\right\rangle+2 h_{j}^{l} h_{i l}\langle\bar{\nabla} \omega, v\rangle .
\end{aligned}
$$

Proof. It is a combination of (2-7), the first of (2-10), (3-3), and (3-5).

Proof of Theorem 1.4. Applying the maximum principle for tensors [Hamilton 1982] to Equation (3-6), we see that the positivity of $\left\{h_{i j}\right\}$ is preserved by the flow (1-1)' if the term $\nabla_{i} \bar{\nabla}^{2} \omega \equiv 0$. This means that the surface $M_{t}$ is always convex along the flow if $\bar{\nabla}^{3} \omega \equiv 0$ and $M_{0}$ is convex. 
Lemma 3.3. Suppose that $M_{t}$ is the solution of $(1-1)^{\prime}$ on $[0, T)$ and the assumptions (i), (ii), and (iii)' in Theorem 1.2 are satisfied. Then $|A|^{2}<C$ on $M_{t}$ for all $t \in[0, T)$.

Proof. Taking a local orthonormal basis $e_{i}$ for $i=1 \ldots n$ on $M_{t}$, Equation (3-1) gives

$$
\begin{aligned}
\frac{d|A|^{2}}{d t} \leq \triangle|A|^{2}+2|A|^{4}-2 h_{i j}\left(\nabla_{i} \bar{\nabla}^{2} \omega\right)\left(e_{j},\right. & v)+2|A|^{2} \bar{\nabla}^{2} \omega(v, v) \\
& -4 h_{i j} h_{j l} \bar{\nabla}^{2} \omega\left(e_{i}, e_{l}\right)-\left\langle\bar{\nabla} \omega, \nabla|A|^{2}\right\rangle .
\end{aligned}
$$

This, together with assumption (i), implies

$$
\begin{aligned}
\frac{d|A|^{2}}{d t} \leq \triangle|A|^{2}+2|A|^{4}+2|A| C_{3}+ & 2|A|^{2} \bar{\lambda} \\
& -4 h_{i j} h_{j l} \bar{\nabla}^{2} \omega\left(e_{i}, e_{l}\right)-\left\langle\bar{\nabla} \omega, \nabla|A|^{2}\right\rangle
\end{aligned}
$$

Next, we estimate $-4 h_{i j} h_{j l} \bar{\nabla}^{2} \omega\left(e_{i}, e_{l}\right)$. Since

$$
\begin{aligned}
-4 h_{i j} h_{j l} \bar{\nabla}^{2} \omega\left(e_{i}, e_{l}\right) & =-4 h_{i j} h_{j l}\left(\bar{\lambda} E\left(e_{i}, e_{l}\right)+\bar{\nabla}^{2} \omega\left(e_{i}, e_{l}\right)-\bar{\lambda} E\left(e_{i}, e_{l}\right)\right) \\
& =-4|A|^{2} \bar{\lambda}-4 h_{i j} h_{j l}\left(\bar{\nabla}^{2} \omega-\bar{\lambda} E\right)\left(e_{i}, e_{l}\right),
\end{aligned}
$$

where $E$ is the unit matrix, we have

$$
\begin{aligned}
-4 h_{i j} h_{j l} \bar{\nabla}^{2} \omega\left(e_{i}, e_{l}\right) & \leq-4|A|^{2} \bar{\lambda}+4\left|h_{i j} h_{j l}\right|\left|\left(\bar{\nabla}^{2} \omega-\bar{\lambda} E\right)\left(e_{i}, e_{l}\right)\right| \\
& \leq-4|A|^{2} \bar{\lambda}+4(\bar{\lambda}-\underline{\lambda})|A|^{2} \\
& =-4 \underline{\lambda}|A|^{2} .
\end{aligned}
$$

Therefore (3-7) becomes

$$
\frac{d|A|^{2}}{d t} \leq \Delta|A|^{2}+2|A|^{4}+2|A| C_{3}+2(\bar{\lambda}-2 \underline{\lambda})|A|^{2}-\left\langle\bar{\nabla} \omega, \nabla|A|^{2}\right\rangle .
$$

Now assumption (iii) ${ }^{\prime}$ means that $C$ satisfies

$$
2 C^{2}+2 C^{1 / 2} C_{3}+2(\bar{\lambda}-2 \underline{\lambda}) C<0,
$$

which, by the continuity of the limit, implies

$$
2 a^{2}+2 \sqrt{a} C_{3}+2(\bar{\lambda}-2 \underline{\lambda}) a \leq 0
$$

for all $a \in(C-\delta, C)$ and for some $\delta \in(0, C)$. Using (3-9) and Hamilton's maximum principle one easily obtains that $|A|^{2}<C$ on $M_{t}$ for all $t \in[0, T)$. Otherwise, by assumption (ii), we can choose the first time $t_{0}$ such that $a\left(t_{0}\right)=C$, where $a(t) \equiv \max _{M_{t}}|A|^{2}$. Then there exists a time $t_{1}<t_{0}$ such that $C-\delta<a(t)<C$ for $t \in\left[t_{1}, t_{0}\right)$, and so $a\left(t_{1}\right)<C$. Hence (3-9) yields

$$
2 a^{2}(t)+2 \sqrt{a(t)} C_{3}+2(\bar{\lambda}-2 \underline{\lambda}) a(t) \leq 0 \quad \text { for all } t \in\left[t_{1}, t_{0}\right) .
$$


Therefore, applying Hamilton's maximum principle [1982] to Equation (3-8) on the time interval $\left[t_{1}, t_{0}\right]$, we have $a(t) \leq a\left(t_{1}\right)<C$ for all $t \in\left[t_{1}, t_{0}\right)$. This contradicts $a\left(t_{0}\right)=C$.

Remark 3.4. The convexity condition on $\omega$, as in assumption (i), and the condition of initially small $|A|^{2}$, as in assumptions (ii) and (iii)', are necessary. In fact, if $\bar{\nabla} \omega \equiv c x$ with either $c<0$, or $c>0$ and $|A|^{2}>c$ on $M_{0}$, we have proved in [Liu and Jian 2007] that $|A|^{2}$ must blow up in finite time, and the flow exists only for a finite time. For this special example, assumption (i) is equivalent to $c>0$ and assumptions (ii) and (iii) ${ }^{\prime}$ are equivalent to $|A|^{2}<c$. This shows the assumptions for Theorem 1.2 are almost optimal.

Proof of Theorem 1.2. From Lemma 3.3 we see that $|A|^{2}$ is bounded uniformly if assumptions (i), (ii), and (iii) ${ }^{\prime}$ are satisfied. Thus, if we can prove that $\left|\nabla^{m} A\right|^{2} \leq C_{m}$ is bounded when $t \rightarrow T$, then by a well-known theorem of partial differential equations, the flow (1-1)' can be extended to $[0, T+\varepsilon$ ) for some small $\varepsilon>0$, where $T<\infty$ is the maximal time interval for which (1-1)' has a smooth solution. This shows that the maximum time interval must be $[0, \infty)$.

To estimate $|\nabla A|^{2}$, the boundedness of $\left|\bar{\nabla}^{4} \omega\right|$ is necessary but is not enough, because we want to calculate the time derivative of $\Gamma_{i j}^{k}$. Because the connection is not a tensor, but the difference of two connections is, $d \Gamma_{i j}^{k} / d t$ is a tensor. Adopting normal coordinates and using the first of (2-9), we have

$$
\begin{aligned}
\frac{d \Gamma_{i j}^{k}}{d t} & =\frac{1}{2} \frac{d}{d t}\left(g^{l k}\left(\frac{\partial g_{i l}}{\partial x_{j}}+\frac{\partial g_{j l}}{\partial x_{i}}-\frac{\partial g_{i j}}{\partial x_{l}}\right)\right) \\
& =\frac{1}{2}\left(g^{l k}\left(\frac{\partial}{\partial x_{i}}\left(\frac{d}{d t} g_{j l}\right)+\frac{\partial}{\partial x_{j}}\left(\frac{d}{d t} g_{i l}\right)-\frac{\partial}{\partial x_{l}}\left(\frac{d}{d t} g_{i j}\right)\right)\right) \\
& =-g^{l k}\left(\frac{\partial}{\partial x_{i}}\left(f h_{j l}\right)+\frac{\partial}{\partial x_{j}}\left(f h_{i l}\right)-\frac{\partial}{\partial x_{l}}\left(f h_{i j}\right)\right) .
\end{aligned}
$$

Noting that $\partial_{i} f=\partial_{i} H-\bar{\nabla}^{2} \omega\left(\partial_{i}, v\right)-h_{i l}\left\langle\bar{\nabla} \omega, \partial_{l}\right\rangle$ and repeating the arguments of Huisken [1984], we obtain the following result.

Lemma 3.5. Suppose that $M_{t}$ is the solution of $(1-1)^{\prime}$ on $[0, T)$ for $T<\infty$. If assumptions (i), (ii), and (iii)' of Theorem 1.2 are satisfied and $\left|\bar{\nabla}^{i} \omega\right|$ is uniformly bounded on $M_{t}$ for $i=1, \ldots, m$, then $\left|\nabla^{m-3} A\right|^{2}$ is uniformly bounded on $M_{t}$.

Using Lemma 3.5, we complete the proof of Theorem 1.2.

Proof of Corollary 1.5. For the special case $\omega=\frac{1}{2} c_{1} x_{1}^{2}+\ldots+\frac{1}{2} c_{n+1} x_{n+1}^{2}$, we have

$$
\bar{\nabla} \omega=\left(c_{1} x_{1}, \ldots, c_{n+1} x_{n+1}\right), \quad \bar{\nabla}^{2} \omega=\left(c_{i} \delta_{i j}\right), \quad \bar{\nabla}^{3} \omega=0 .
$$

Let $M=\max c_{i}$, and $m=\min c_{i}$. Applying Lemma 3.3 we find, if $M<2 m$ and $|A|^{2}<2 m-M$ on $M_{0}$, that $|A|^{2}<2 m-M$ as long as flow (1-1)' exists. To get the 
long-time existence we must estimate the higher derivatives of $|A|^{2}$. But Lemma 3.5 can not be applied directly, because $|\bar{\nabla} \omega|$ may turn to be infinite if the surface expands to infinity. However, we can prove that the surface will not expand to infinity in finite time. First we need a theorem.

Lemma 3.6 [Schnürer and Smoczyk 2002]. Let $F$ be a smooth immersed solution of (1-1)', and let $\widetilde{F}$ be an immersed solution of this evolution equation. If $\widetilde{F}$ is contained in a connected component of $\mathbb{R}^{n+1} \backslash F$ or in the closure of such a component at the beginning of the evolution, then this remains true during the evolution.

Since $|A|^{2} \leq 2 m-M$ on $M_{0}$, we will prove that if the initial surface is a center sphere, the sphere will expand to infinity as $t \rightarrow \infty$.

Lemma 3.7. Suppose that $M_{0}=S^{n}(R)$ is the initial surface of the flow (1-1)' and $\omega, m$, and $M$ are as above. Let $s(t):=\frac{1}{2}\left|F_{t}\right|^{2}$ where $F_{t}$ is the position vector of $M_{t}$. If $|A|^{2}<2 m-M$ on $M_{0}$, then $\widetilde{C} \equiv(2 m s(0)-n)>0$ and $s \geq((n+\widetilde{C}) /(2 m)) e^{2 m t}$ for all $t>0$.

Proof. Note that

$$
\frac{d s}{d t}=\left\langle\frac{d F}{d t}, F\right\rangle=-(H-\langle\bar{\nabla} \omega, v\rangle)\langle F, v\rangle=-n+\langle\bar{\nabla} \omega, v\rangle\langle F, v\rangle .
$$

Since $v=F /|F|$ holds on the spheres, we have

$$
\langle\bar{\nabla} \omega, v\rangle=\frac{1}{|F|}\langle\bar{\nabla} \omega, F\rangle=\frac{1}{|F|}\left(c_{1} F_{1}^{2}+\ldots+c_{n+1} F_{n+1}^{2}\right) \geq \frac{1}{|F|} m|F|^{2}=m|F| .
$$

Hence $d s / d t \geq-n+2 m s$. Therefore $s \geq((n+\widetilde{C}) /(2 m)) e^{2 m t}$ for all $t>0$ if $\widetilde{C}>0$. Now by the initial condition, we have

$$
2 m-M>|A|^{2}=\frac{1}{n} H^{2}=\frac{1}{n} \frac{n^{2}}{|F|^{2}}=\frac{n}{2 s(0)},
$$

which implies $2 s>n /(2 m-M)$ and $\widetilde{C}>0$. This proves Lemma 3.7.

So for initial hypersurface $M^{n}$, we can find a large enough center sphere to contain it, while $|A|^{2}<2 m-M$ is satisfied on the sphere. As a consequence Lemma 3.6 and Lemma 3.7, imply that $M_{t}$ will not expand to infinity in finite time. This with the above discussion finishes the proof of Corollary 1.5.

\section{Higher codimension case}

Here we will prove Theorem 1.1. As the hypersurface case, the key step is to derive the evolution equation of $|A|^{2}$. For this purpose, we want to calculate the evolution equation of the second fundamental form tensor. In the following, for $x \in M^{n}$ we take an orthonormal basis $e_{1}, \cdots, e_{n}, e_{n+1}, \cdots, e_{n+k}$ of $\mathbb{R}^{n+k}$ such 
that $\left\{e_{1}, \cdots, e_{n}\right\}$ is a basis of $T_{x} M^{n}$ and $\left\{e_{n+1}, \cdots, e_{n+k}\right\}$ (denoted by $\left\{e_{\alpha}\right\}$ ) is the unit normal vector.

Proposition 4.1. Suppose flow (1-1) holds for $t \in[0, T)$ with $T \leq \infty$. Then we have these equations on $M_{t}$ for all $t \in[0, T)$ :

$$
\begin{aligned}
\frac{d h_{\alpha i j}}{d t}-\Delta h_{\alpha i j}= & -H_{\beta} h_{\beta i l} h_{\alpha j l}-\left(\nabla_{j} \bar{\nabla}^{2} \omega\right)\left(e_{i}, e_{\alpha}\right)+h_{\beta i j} \bar{\nabla}^{2} \omega\left(e_{\beta}, e_{\alpha}\right) \\
& -h_{\alpha k j} \bar{\nabla}^{2} \omega\left(e_{i}, e_{k}\right)-h_{\alpha i k} \bar{\nabla}^{2} \omega\left(e_{j}, e_{k}\right)+h_{\beta i j}\left\langle e_{\beta}, \frac{d e_{\alpha}}{d t}\right\rangle \\
+ & \left\langle\bar{\nabla} \omega, e_{\beta}\right\rangle\left(h_{\beta i k} h_{\alpha j k}+h_{\beta j k} h_{\alpha i k}\right)-\left\langle\bar{\nabla} \omega, \nabla h_{\alpha i j}\right\rangle \\
- & h_{\alpha i m}\left(h_{\gamma m j} h_{\gamma k k}-h_{\gamma m k} h_{\gamma k j}\right)-h_{\alpha m k}\left(h_{\gamma m j} h_{\gamma i k}-h_{\gamma m k} h_{\gamma i j}\right) \\
- & h_{\beta i k}\left(-h_{\beta k m} h_{\alpha j m}+h_{\beta j m} h_{\alpha k m}\right) .
\end{aligned}
$$

Proof. Because both sides are tensorial, we may calculate in normal coordinates. Since $\nabla_{j} \nabla_{i} F=-h_{\alpha i j} e_{\alpha}$, then by flow (1-1) we have

$$
\begin{aligned}
\frac{d h_{\alpha i j}}{d t} & =-\frac{d}{d t}\left\langle\nabla_{j} \nabla_{i} F, e_{\alpha}\right\rangle \\
& =-\left\langle\nabla_{j} \nabla_{i}\left(-H_{\beta} e_{\beta}+\omega_{\beta} e_{\beta}\right), e_{\alpha}\right\rangle-\left\langle\nabla_{j} \nabla_{i} F, \frac{d e_{\alpha}}{d t}\right\rangle \\
& =\left\langle\nabla_{j} \nabla_{i}\left(H_{\beta} e_{\beta}\right), e_{\alpha}\right\rangle-\left\langle\nabla_{j} \nabla_{i}\left(\omega_{\beta} e_{\beta}\right), e_{\alpha}\right\rangle+h_{\beta i j}\left\langle e_{\beta}, \frac{d e_{\alpha}}{d t}\right\rangle .
\end{aligned}
$$

By Weingarten Equation (2-2), we have

$$
\begin{aligned}
\nabla_{j} \nabla_{i} e_{\beta} & =\left(\nabla_{j} h_{\beta i l}\right) e_{l}-h_{\beta i l} h_{\gamma j l} e_{\gamma}+\left(\nabla_{j} C_{i \beta}^{\gamma}\right) e_{\gamma}+C_{i \beta}^{\gamma} \nabla_{j} e_{\gamma} \\
& =\left(\nabla_{j} h_{\beta i l}\right) e_{l}-h_{\beta i l} h_{\gamma j l} e_{\gamma}+\left(\nabla_{j} C_{i \beta}^{\gamma}\right) e_{\gamma}+C_{i \beta}^{\gamma} h_{\gamma j l} e_{l}+C_{i \beta}^{\gamma} C_{j \gamma}^{\eta} e_{\eta} \\
& =h_{\beta i l, j} e_{l}-h_{\beta i l} h_{\gamma j l} e_{\gamma}+\left(\nabla_{j} C_{i \beta}^{\gamma}\right) e_{\gamma}+C_{i \beta}^{\gamma} C_{j \gamma}^{\eta} e_{\eta} .
\end{aligned}
$$

This, together with (2-2), implies

$$
\begin{aligned}
\nabla_{j} \nabla_{i}\left(H_{\beta} e_{\beta}\right)= & \left(\nabla_{j} \nabla_{i} H_{\beta}\right) e_{\beta}+\left(\nabla_{j} H_{\beta}\right) \nabla_{i} e_{\beta}+\left(\nabla_{i} H_{\beta}\right) \nabla_{j} e_{\beta}+H_{\beta} \nabla_{j} \nabla_{i} e_{\beta} \\
= & \left(\nabla_{j} \nabla_{i} H_{\beta}\right) e_{\beta}+\left(\nabla_{j} H_{\beta}\right) h_{\beta i l} e_{l}+\left(\nabla_{j} H_{\beta}\right) C_{i \beta}^{\gamma} e_{\gamma} \\
& +\left(\nabla_{i} H_{\beta}\right) h_{\beta j l} e_{l}+\left(\nabla_{i} H_{\beta} C_{j \beta}^{\gamma}\right) e_{\gamma}+H_{\beta} \nabla_{j} \nabla_{i} e_{\beta} .
\end{aligned}
$$

Hence,

$$
\begin{aligned}
\left\langle\nabla_{j} \nabla_{i}\left(H_{\beta} e_{\beta}\right), e_{\alpha}\right\rangle=\nabla_{j} \nabla_{i} H_{\alpha}+\nabla_{j} & H_{\beta} C_{i \beta}^{\alpha}+\nabla_{i} H_{\beta} C_{j \beta}^{\alpha} \\
& -H_{\beta} h_{\alpha j l} h_{\beta i l}+H_{\beta} \nabla_{j} C_{i \beta}^{\alpha}+H_{\beta} C_{i \beta}^{\gamma} C_{j \gamma}^{\alpha} .
\end{aligned}
$$

Note that

$$
\begin{aligned}
\sum_{k} h_{\alpha k k, i j}=\nabla_{j} \nabla_{i} H_{\alpha}+\nabla_{j} H_{\beta} C_{i \beta}^{\alpha}+\nabla_{i} H_{\beta} C_{j \beta}^{\alpha} & \\
& +H_{\beta} \nabla_{j} C_{i \beta}^{\alpha}+H_{\beta} C_{i \beta}^{\gamma} C_{j \gamma}^{\alpha}-2 h_{\alpha k l} \frac{\partial \Gamma_{i k}^{l}}{\partial x_{j}},
\end{aligned}
$$


the last term of which is zero because $\Gamma_{i k}^{l}=-\Gamma_{i l}^{k}$. Then we use this equation to rewrite (4-5) as

$$
\left\langle\nabla_{j} \nabla_{i}\left(H_{\beta} e_{\beta}\right), e_{\alpha}\right\rangle=\sum_{k} h_{\alpha k k, i j}-H_{\beta} h_{\alpha j l} h_{\beta i l} .
$$

Simon's identity gives

$$
\sum_{k} h_{\alpha k k, i j}=\triangle h_{\alpha i j}-\left(h_{\beta i k} R_{\beta \alpha j k}^{\perp}+h_{\alpha m k} R_{m i j k}+h_{\alpha i m} R_{m k j k}\right) .
$$

Putting (4-7) in (4-6) and using (2-1), we obtain

$$
\begin{array}{r}
\left\langle\nabla_{j} \nabla_{i}\left(H_{\beta} e_{\beta}\right), e_{\alpha}\right\rangle=\Delta h_{\alpha i j}-H_{\beta} h_{\alpha j l} h_{\beta i l}-h_{\alpha i m}\left(h_{\gamma m j} h_{\gamma k k}-h_{\gamma m k} h_{\gamma k j}\right) \\
-h_{\alpha m k}\left(h_{\gamma m j} h_{\gamma i k}-h_{\gamma m k} h_{\gamma i j}\right)-h_{\beta i k}\left(-h_{\beta k m} h_{\alpha j m}+h_{\beta j m} h_{\alpha k m}\right)
\end{array}
$$

Next, we use (2-2) to calculate the term $\nabla_{j} \nabla_{i}\left(\omega_{\beta} e_{\beta}\right)$ in (4-2). Since

$$
\begin{aligned}
\nabla_{j} \nabla_{i}\left(\omega_{\beta} e_{\beta}\right)= & \nabla_{j} \nabla_{i}\left(\bar{\nabla} \omega-\left\langle\bar{\nabla} \omega, e_{k}\right\rangle e_{k}\right) \\
= & \nabla_{j} \nabla_{i} \bar{\nabla} \omega-\nabla_{j}\left(\bar{\nabla}^{2} \omega\left(e_{i}, e_{k}\right) e_{k}\right. \\
& \left.-h_{\beta i k}\left\langle\bar{\nabla} \omega, e_{\beta}\right\rangle e_{k}-h_{\beta i k}\left\langle\bar{\nabla} \omega, e_{k}\right\rangle e_{\beta}\right) \\
= & \nabla_{j} \nabla_{i} \bar{\nabla} \omega-\nabla_{j}\left(\bar{\nabla}^{2} \omega\left(e_{i}, e_{k}\right)\right) e_{k}+h_{\beta j k} \bar{\nabla}^{2} \omega\left(e_{i}, e_{k}\right) e_{\beta} \\
& +\nabla_{j}\left(h_{\beta i k}\left\langle\bar{\nabla} \omega, e_{\beta}\right\rangle\right) e_{k}-h_{\beta i k} h_{\gamma j k}\left\langle\bar{\nabla} \omega, e_{\beta}\right\rangle e_{\gamma} \\
& +\nabla_{j}\left(h_{\beta i k}\right)\left\langle\bar{\nabla} \omega, e_{k}\right\rangle e_{\beta}+h_{\beta i k} \bar{\nabla}^{2} \omega\left(e_{j}, e_{k}\right) e_{\beta} \\
& -h_{\beta i k} h_{\gamma j k}\left\langle\bar{\nabla} \omega, e_{\gamma}\right\rangle e_{\beta}+h_{\beta i k}\left\langle\bar{\nabla} \omega, e_{k}\right\rangle \nabla_{j} e_{\beta},
\end{aligned}
$$

we have

$$
\begin{aligned}
\left\langle\nabla_{j} \nabla_{i}\left(\omega_{\beta} e_{\beta}\right), e_{\alpha}\right\rangle= & \left\langle\nabla_{j} \nabla_{i} \bar{\nabla} \omega, e_{\alpha}\right\rangle+h_{\alpha j k} \bar{\nabla}^{2} \omega\left(e_{i}, e_{k}\right)+h_{\alpha i k} \bar{\nabla}^{2} \omega\left(e_{j}, e_{k}\right) \\
& -\left\langle\bar{\nabla} \omega, e_{\beta}\right\rangle\left(h_{\beta i k} h_{\alpha j k}+h_{\alpha i k} h_{\beta j k}\right) \\
& +\left(\nabla_{j} h_{\alpha i k}+C_{j \beta}^{\alpha} h_{\beta i k}\right)\left\langle\bar{\nabla} \omega, e_{k}\right\rangle \\
= & \left\langle\nabla_{j} \nabla_{i} \bar{\nabla} \omega, e_{\alpha}\right\rangle+h_{\alpha j k} \bar{\nabla}^{2} \omega\left(e_{i}, e_{k}\right)+h_{\alpha i k} \bar{\nabla}^{2} \omega\left(e_{j}, e_{k}\right) \\
& -\left\langle\bar{\nabla} \omega, e_{\beta}\right\rangle\left(h_{\beta i k} h_{\alpha j k}+h_{\alpha i k} h_{\beta j k}\right)+h_{\alpha i k, j}\left\langle\bar{\nabla} \omega, e_{k}\right\rangle .
\end{aligned}
$$

Due to the Codazzi equation (2-3), we have

$$
\begin{aligned}
\left\langle\nabla_{j} \nabla_{i}\left(\omega_{\beta} e_{\beta}\right), e_{\alpha}\right\rangle= & \left\langle\nabla_{j} \nabla_{i} \bar{\nabla} \omega, e_{\alpha}\right\rangle+h_{\alpha j k} \bar{\nabla}^{2} \omega\left(e_{i}, e_{k}\right)+h_{\alpha i k} \bar{\nabla}^{2} \omega\left(e_{j}, e_{k}\right) \\
& -\left\langle\bar{\nabla} \omega, e_{\beta}\right\rangle\left(h_{\beta i k} h_{\alpha j k}+h_{\alpha i k} h_{\beta j k}\right)+\left\langle\bar{\nabla} \omega, \nabla h_{\alpha i j}\right\rangle \\
= & \left(\nabla_{j} \bar{\nabla} \omega^{2}\right)\left(e_{i}, e_{\alpha}\right)-h_{\beta i j} \bar{\nabla}^{2} \omega\left(e_{\alpha}, e_{\beta}\right) \\
& +h_{\alpha j k} \bar{\nabla}^{2} \omega\left(e_{i}, e_{k}\right)+h_{\alpha i k} \bar{\nabla}^{2} \omega\left(e_{j}, e_{k}\right) \\
& -\left\langle\bar{\nabla} \omega, e_{\beta}\right\rangle\left(h_{\beta i k} h_{\alpha j k}+h_{\alpha i k} h_{\beta j k}\right)+\left\langle\bar{\nabla} \omega, \nabla h_{\alpha i j}\right\rangle .
\end{aligned}
$$


Now (4-1) follows from (4-2), (4-8), and (4-9).

Proposition 4.2. Suppose flow (1-1) holds for $t \in[0, T)$ with $T \leq \infty$. Then we have this equation on $M_{t}$ for all $t \in[0, T)$ :

$$
\begin{aligned}
\frac{d|A|^{2}}{d t}= & \Delta|A|^{2}-2|\nabla A|^{2}-2 h_{\alpha i j}\left(\nabla_{j} \bar{\nabla}^{2} \omega\right)\left(e_{i}, e_{\alpha}\right) \\
& +2 h_{\alpha i j} h_{\beta i j} \bar{\nabla}^{2} \omega\left(e_{\alpha}, e_{\beta}\right)-4 h_{\alpha i k} h_{\alpha i j} \bar{\nabla}^{2} \omega\left(e_{j}, e_{k}\right)-\left\langle\bar{\nabla} \omega, \nabla|A|^{2}\right\rangle \\
& +2 \sum_{\alpha, \gamma, i, m}\left(\sum_{k} h_{\alpha i k} h_{\gamma m k}-h_{\alpha m k} h_{\gamma i k}\right)^{2}+2 \sum_{i, j, k, m}\left(\sum_{\alpha} h_{\alpha i j} h_{\alpha m k}\right)^{2} .
\end{aligned}
$$

Proof. We calculate in normal coordinates. Because $|A|^{2}=g^{i j} g^{k l} h_{\alpha i k} h_{\alpha l j}$,

$$
\frac{d|A|^{2}}{d t}=2 \frac{d g^{i k}}{d t} h_{\alpha i j} h_{\alpha k j}+2 \frac{d h_{\alpha i j}}{d t} h_{\alpha i j} .
$$

Hence by the second of (2-9), (4-1), and (4-11), we have

$$
\begin{aligned}
\frac{d|A|^{2}}{d t}= & 2 h_{\alpha i j} \triangle h_{\alpha i j}+4\left(H_{\beta}-\omega_{\beta}\right) h_{\beta i k} h_{\alpha i j} h_{\alpha k j}-2 H_{\beta} h_{\alpha i j} h_{\beta i l} h_{\alpha j l} \\
& -2 h_{\alpha i j}\left(\nabla_{j} \bar{\nabla}^{2} \omega\right)\left(e_{i}, e_{\alpha}\right)+2 h_{\alpha i j} h_{\beta i j} \bar{\nabla}^{2} \omega\left(e_{\beta}, e_{\alpha}\right) \\
& -4 h_{\alpha i j} h_{\alpha k j} \bar{\nabla}^{2} \omega\left(e_{i}, e_{k}\right)+2 h_{\alpha i j} h_{\beta i j}\left\langle e_{\beta}, \frac{d e_{\alpha}}{d t}\right\rangle+4 h_{\alpha i j} \omega_{\beta} h_{\beta i k} h_{\alpha j k} \\
& -\left\langle\bar{\nabla} \omega, \nabla|A|^{2}\right\rangle-2 h_{\alpha i j} h_{\alpha i m} h_{\gamma m j} H_{\gamma}+2 h_{\alpha i j} h_{\alpha i m} h_{\gamma m k} h_{\gamma k j} \\
& -2 h_{\alpha i j} h_{\alpha m k}\left(h_{\gamma m j} h_{\gamma i k}-h_{\gamma m k} h_{\gamma i j}\right)-2 h_{\alpha i j} h_{\beta i k}\left(h_{\beta l j} h_{\alpha l k}-h_{\beta l k} h_{\alpha l j}\right) .
\end{aligned}
$$

Observing that $2 h_{\alpha i j} h_{\beta i j}\left\langle e_{\beta}, d e_{\alpha} / d t\right\rangle$ is zero by symmetry and that $2 h_{\alpha i j} \Delta h_{\alpha i j}=$ $\triangle|A|^{2}-2|\nabla A|^{2}$, we have

$$
\begin{aligned}
\frac{d|A|^{2}}{d t}= & \Delta|A|^{2}-2|\nabla A|^{2}-2 h_{\alpha i j}\left(\bar{\nabla}_{j} \bar{\nabla}^{2} \omega\right)\left(e_{i}, e_{\alpha}\right)+2 h_{\alpha i j} h_{\beta i j} \bar{\nabla}^{2} \omega\left(e_{\beta}, e_{\alpha}\right) \\
& -4 h_{\alpha i j} h_{\alpha k j} \bar{\nabla}^{2} \omega\left(e_{i}, e_{k}\right)-\left\langle\bar{\nabla} \omega, \nabla|A|^{2}\right\rangle+2 h_{\alpha i j} h_{\alpha i m} h_{\gamma m k} h_{\gamma k j} \\
& -2 h_{\alpha i j} h_{\alpha m k}\left(h_{\gamma m j} h_{\gamma i k}-h_{\gamma m k} h_{\gamma i j}\right)-2 h_{\alpha i j} h_{\beta i k}\left(h_{\beta l j} h_{\alpha l k}-h_{\beta l k} h_{\alpha l j}\right) .
\end{aligned}
$$

But the last three terms can be calculated as follows:

$2 h_{\alpha i j} h_{\alpha i m} h_{\gamma m k} h_{\gamma k j}-2 h_{\alpha i j} h_{\alpha m k} h_{\gamma m j} h_{\gamma i k}$

$$
\begin{gathered}
+2 h_{\alpha i j} h_{\alpha m k} h_{\gamma m k} h_{\gamma i j}-2 h_{\alpha i j} h_{\beta i k}\left(h_{\beta l j} h_{\alpha l k}-h_{\beta l k} h_{\alpha l j}\right) \\
=4 h_{\alpha i j} h_{\alpha i m} h_{\gamma m k} h_{\gamma k j}-4 h_{\alpha i j} h_{\alpha m k} h_{\gamma m j} h_{\gamma i k}+2 h_{\alpha i j} h_{\gamma m k} h_{\alpha m k} h_{\gamma i j} .
\end{gathered}
$$

Since

$2 h_{\alpha i j} h_{\alpha i m} h_{\gamma m k} h_{\gamma k j}-2 h_{\alpha i j} h_{\alpha m k} h_{\gamma m j} h_{\gamma i k}$

$$
=2 h_{\alpha i j} h_{\alpha i k} h_{\gamma m k} h_{\gamma m j}-2 h_{\alpha i j} h_{\alpha m k} h_{\gamma m j} h_{\gamma i k}
$$




$$
\begin{aligned}
& =2 h_{\alpha i j} h_{\gamma m j}\left(h_{\alpha i k} h_{\gamma m k}-h_{\alpha m k} h_{\gamma i k}\right) \\
& =h_{\alpha i j} h_{\gamma m j}\left(h_{\alpha i k} h_{\gamma m k}-h_{\alpha m k} h_{\gamma i k}\right)+h_{\alpha m j} h_{\gamma i j}\left(h_{\alpha m k} h_{\gamma i k}-h_{\alpha m k} h_{\alpha i k}\right) \\
& =\sum_{\alpha, \gamma, i, m}\left(\sum_{k} h_{\alpha i k} h_{\gamma m k}-h_{\alpha m k} h_{\gamma i k}\right)^{2}
\end{aligned}
$$

and $2 h_{\alpha i j} h_{\gamma m k} h_{\alpha m k} h_{\gamma i j}=2 \sum_{i, j, k, m}\left(\sum_{\alpha} h_{\alpha i j} h_{\alpha m k}\right)^{2}$, we are done.

Lemma 4.3. Suppose that $M_{t}$ is the solution of (1-1) on $[0, T)$ and that assumptions (i), (ii) and (iii) of Theorem 1.1 hold. Then $|A|^{2}<C$ on $M_{t}$ for all $t \in[0, T)$.

Proof. The proof is almost the same as that of Lemma 3.3 in the case of hypersurfaces. It follows from Schwarz inequality that

$$
\begin{array}{r}
2 \sum_{i, j, k, m}\left(\sum_{\alpha} h_{\alpha i j} h_{\alpha m k}\right)^{2} \leq 2 \sum_{i, j, k, m}\left(\sum_{\alpha} h_{\alpha i j}^{2}\right)\left(\sum_{\alpha} h_{\alpha m k}^{2}\right)=2|A|^{4}, \\
\sum_{\alpha, \gamma, i, m}\left(\sum_{k} h_{\alpha i k} h_{\gamma m k}-h_{\alpha m k} h_{\gamma i k}\right)^{2} \leq 4 \sum_{\alpha, \gamma, i, m}\left(\sum_{k} h_{\alpha i k} h_{\gamma m k}\right)^{2} \leq 4|A|^{4} .
\end{array}
$$

Consequently, using the same technique as from (3-7) to (3-8), we obtain

$$
\frac{d|A|^{2}}{d t} \leq \triangle|A|^{2}-\left\langle\bar{\nabla} \omega, \nabla|A|^{2}\right\rangle+10|A|^{4}+2|A| C_{3}+2(\bar{\lambda}-2 \underline{\lambda})|A|^{2} .
$$

The result follows by the arguments below (3-8) of Lemma 3.3.

Proof of Theorem 1.1. Using Lemma 4.3 and repeating the proof of Theorem 1.2, one easily proves Theorem 1.1 .

\section{References}

[Chapman and Richardson 1997] S. J. Chapman and G. Richardson, "Vortex pinning by inhomogeneities in type-II superconductors”, Phys. D 108:4 (1997), 397-407. MR 98k:82201 Zbl 1039. 82510

[Chapman et al. 1996] S. J. Chapman, Q. Du, and M. D. Gunzburger, "A model for variable thickness superconducting thin films", Z. Angew. Math. Phys. 47:3 (1996), 410-431. MR 97f:82048 Zbl 0862.35119

[Chen and Li 2001] J. Chen and J. Li, "Mean curvature flow of surface in 4-manifolds", Adv. Math. 163:2 (2001), 287-309. MR 2002h:53116 Zbl 1002.53046

[Chen and Li 2004] J. Chen and J. Li, "Singularity of mean curvature flow of Lagrangian submanifolds”, Invent. Math. 156:1 (2004), 25-51. MR 2004m:53116 Zbl 1059.53052

[Hamilton 1982] R. S. Hamilton, "Three-manifolds with positive Ricci curvature", J. Differential Geom. 17:2 (1982), 255-306. MR 84a:53050 Zbl 0504.53034

[Hamilton 1996] R. Hamilton, "Lectures on geometric evolution equations", Lecture notes, 1996.

[Huisken 1984] G. Huisken, "Flow by mean curvature of convex surfaces into spheres", J. Differential Geom. 20:1 (1984), 237-266. MR 86j:53097 Zbl 0556.53001

[Huisken 1990] G. Huisken, "Asymptotic behavior for singularities of the mean curvature flow", $J$. Differential Geom. 31:1 (1990), 285-299. MR 90m:53016 Zbl 0694.53005 
[Jerrard and Soner 1999] R. L. Jerrard and H. M. Soner, "Scaling limits and regularity results for a class of Ginzburg-Landau systems", Ann. Inst. H. Poincaré Anal. Non Linéaire 16:4 (1999), 423466. MR 2000f:35135 Zbl 0944.35006

[Jian 2006] H.-Y. Jian, "Translating solitons of mean curvature flow of noncompact spacelike hypersurfaces in Minkowski space”, J. Differential Eq. 220:1 (2006), 147-162. MR 2006i:53095 Zbl 02242182

[Jian and Liu 2006] H. Y. Jian and Y. N. Liu, "Ginzburg-Landau vortex and mean curvature flow with external force field”, Acta Math. Sin. (Engl. Ser.) 22:6 (2006), 1831-1842. MR 2007g:35103 Zbl 1113.35091

[Jian and Song 2001] H.-Y. Jian and B.-H. Song, "Vortex dynamics of Ginzburg-Landau equations in inhomogeneous superconductors", J. Differential Eq. 170:1 (2001), 123-141. MR 2002j:35152 Zbl 0973.35176

[Jian and $\mathrm{Xu}$ 2003] H. Jian and X. Xu, "The vortex dynamics of a Ginzburg-Landau system under pinning effect”, Sci. China Ser. A 46:4 (2003), 488-498. MR 2004i:35165

[Jian et al. 2005] H. Jian, Q. Liu, and X. Chen, "Convexity and symmetry of translating solitons in mean curvature flows", Chinese Ann. Math. Ser. B 26:3 (2005), 413-422. MR 2006e:53120 Zbl 02226984

[Lin 1996] F. H. Lin, "Some dynamical properties of Ginzburg-Landau vortices", Comm. Pure Appl. Math. 49:4 (1996), 323-359. MR 97c:35189 Zbl 0853.35058

[Lin 1998] F. H. Lin, "Complex Ginzburg-Landau equations and dynamics of vortices, filaments, and codimension-2 submanifolds", Comm. Pure Appl. Math. 51:4 (1998), 385-441. MR 98k:35177 Zbl 0932.35121

[Liu and Jian 2007] Y.-n. Liu and H.-y. Jian, "Evolution of hypersurfaces by the mean curvature minus an external force field", Sci. China Ser. A 50:2 (2007), 231-239. MR MR2306089 Zbl 1120.35019

[Schnürer and Smoczyk 2002] O. C. Schnürer and K. Smoczyk, "Evolution of hypersurfaces in central force fields", J. Reine Angew. Math. 550 (2002), 77-95. MR 2004c:53102 Zbl 1019.53033

[Smoczyk and Wang 2002] K. Smoczyk and M.-T. Wang, "Mean curvature flows of Lagrangians submanifolds with convex potentials", J. Differential Geom. 62 (2002), 243-257. MR 2004d:53086 Zbl 1070.53042

[Wang 2002] M.-T. Wang, "Long-time existence and convergence of graphic mean curvature flow in arbitrary codimension”, Invent. Math. 148:3 (2002), 525-543. MR 2003b:53073 Zbl 1039.53072

Received April 26, 2007. Revised September 10, 2007.

HUAI-YU JIAN

DEPARTMENT OF MATHEMATICAL SCIENCES

TSINGHUA UNIVERSITY

BEIJING 100084

CHINA

hjian@math.tsinghua.edu.cn

\section{YANNAN LIU \\ SCHOOL OF Mathematical Sciences \\ BEIJING UNIVERSITY \\ BEIJING 100871 \\ CHINA}

liuyn04@mails.tsinghua.edu.cn 